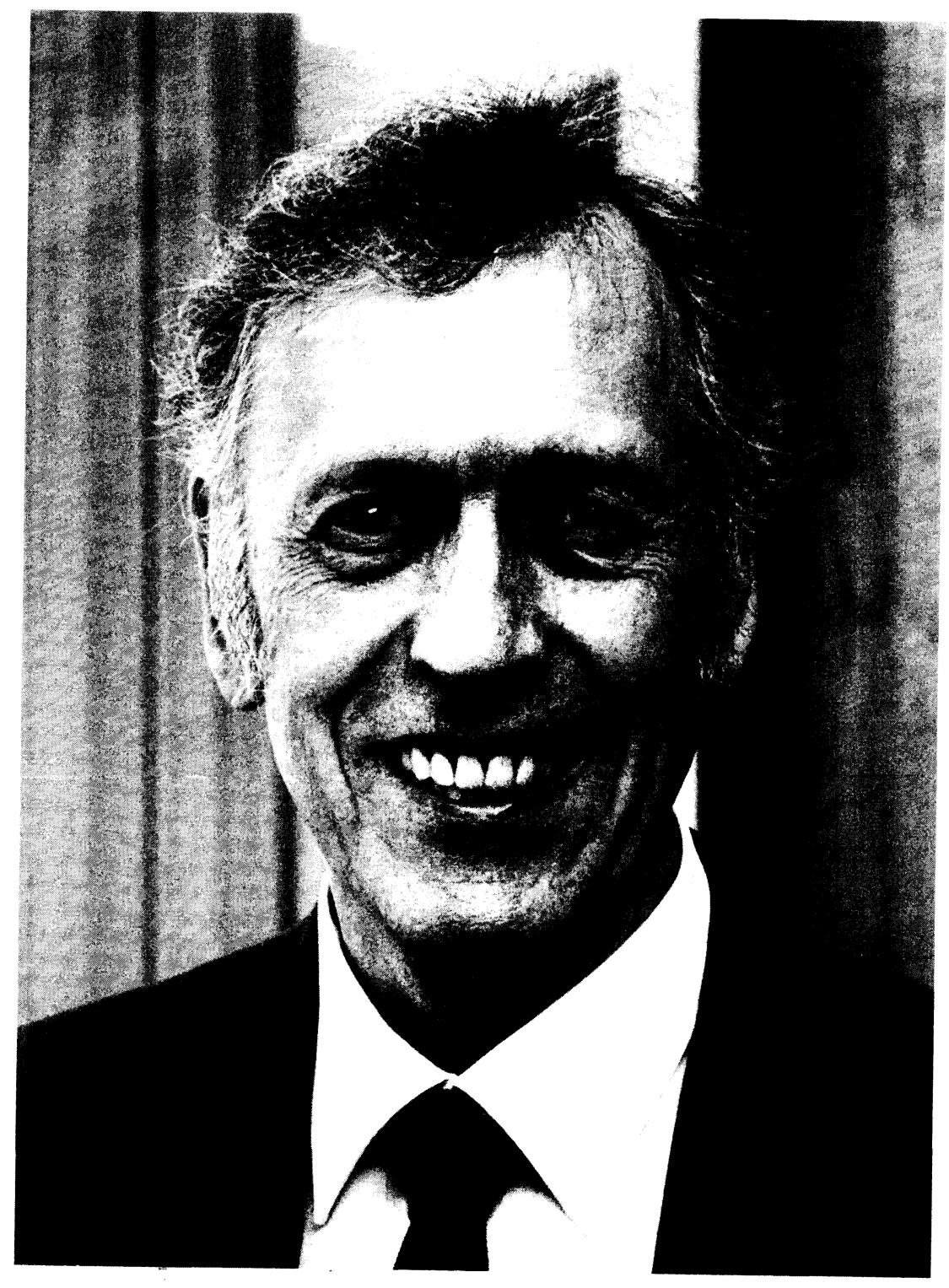

Paul Röhrig

$31 / 51925$ - 24/4 2007 


\section{Paul Röhrig in memoriam}

\section{Von Norbert Vogel}

Um des Menschen willen - diese Grundtvig zugeeignete Leitidee, die Paul Röhrig dem Kölner Grundtvig-Kongress gab, trifft zweifellos auch den Kern seines eigenen Lebenswerks. Humanität war ihm das durchgängige Motiv, das sowohl sein soziales und sozialpolitisches Wirken als auch sein wissenschaftliches Werk bestimmte. Als Arbeiterkind galten ihm Bildung und Aufklärung als maßgebliches Vehikel eines sozialverträglichen gesellschaftlichen Wandels und Ausgleichs. Vor diesem Hintergrund lag es für Paul Röhrig nahe, sich beruflich dem Bildungssystem zu verpflichten.

Dass in Sonderheit die Erwachsenenbildung im Vordergrund stand, verdankt sich aufrüttelnden Kriegserlebnissen im nationalsozialistischen Unrechtsstaat, aber auch prägenden Erfahrungen während eines mehrwöchigen Studienaufenthalts in England im Jahre 1951. Seiner Auffassung, dass eine fortschreitende Demokratisierung im Nachkriegsdeutschland auch und gerade durch eine breite (politische) Bildungsarbeit zu erreichen sei, bot seine Funktion als stellvertretender Direktor an der Volkshochschule Köln, die er in den Jahren von 1954 bis 1970 innehatte, einen fruchtbaren Wirkungsraum. Das vorausgegangene Studium der Pädagogik, Philosophie und Germanistik an der Universität zu Köln, dem 1961 eine Promotion über politische Erwachsenenbildung an der Universität Mainz folgte, schuf die dafür notwendigen Voraussetzungen.

Mit dem 1970 erfolgten Ruf auf einen Lehrstuhl für Allgemeine Pädagogik zunächst an die Pädagogische Hochschule Rheinland, den er nach der späteren Integration in die Universität zu Köln bis zu seiner Emeritierung im Jahre 1990 innehatte, war schließlich der Weg des akademischen Lehrers vorgezeichnet, der seine Praxiserfahrung als Erwachsenenbildner nunmehr in Lehre und Forschung ergänzte und vertiefte. Die dänische Erwachsenenbildung nahm hierbei durchgängig breiten Raum ein. So führte ihn seine Lehrtätigkeit immer wieder nach Dänemark, indem er seine Studierenden auf Exkursionen mit der vielfältigen Praxis der dänischen Volkshochschulen, aber auch mit deren Ideenhintergrund vertraut machte. In einem Fall mündete die nachhaltige Erfahrung von Studierenden unter Paul Röhrigs Regie in der Gründung eines Volkshochschulheims für junge Arbeiter bei der Stadt Köln (Haus Balchem), das über viele Jahre Bestand hatte.

Auch die Forschungsarbeit war in starkem Maße von der Beschäftigung mit der dänischen Volkshochschule bzw. Grundtvig bestimmt. Zahlreiche Veröffentlichungen $\mathrm{zu}$ konzeptionellen, 
bildungspolitischen und didaktischen Aspekten der Grundtvigschen Volkshochschule dokumentieren dies in eindrücklicher Weise. Darüber hinaus prägen neben verschiedenen Abhandlungen $\mathrm{zu}$ allgemeinen Fragen der Pädagogik einschlägige Werke zur Bildungsgeschichte - und hier insbesondere der Erwachsenenbildung - sowie seine namhaften bildungstheoretischen Abhandlungen sein bleibendes wissenschaftliches Oeuvre. Dass im Blickfeld der Betrachtung neben der dänischen Volkshochschule immer auch die Arbeiterbildung stand, erklärt sich aus seiner eigenen Biographie und knüpft zugleich an von ihm immer wieder thematisierte Bestrebungen der Weimarer Volksbildung an, die Volkshochschule in ihren verschiedenen Organisationsformen auch der Arbeiterschicht zugänglich zu machen.

Als einer der Höhepunkte von Röhrigs akademischem Wirken kann sicherlich der internationale Grundtvig-Kongress gelten, der unter seiner Federführung 1988 im Rahmen des 600. Jahrestags der Gründung der Universität zu Köln stattfand. Der 1991 veröffentlichte Kongressband dokumentiert in eindrucksvoller Weise seine Intention, Grundtvigs historisches, theologisches, poetisches und nicht zuletzt sein politisches und pädagogisches Wirken einer breiten interdisziplinären wie internationalen wissenschaftlichen Diskussion zuzuführen. Und in der Tat dürfte es neben der Kopenhagener GrundtvigKonferenz im Jahre 1983 kaum mehr gelungen sein, die internationale Grundtvig-Rezeption unter so breiter Beteiligung von namhaften Grundtvig-Experten zu erörtern, um über eine kritische Standortbestimmung von Grundtvigs Werk und Wirken dessen bleibendes geistiges Erbe für Erwachsenenbildung, Schule, Kirche und soziales Leben herauszuarbeiten. Dass Paul Röhrig 1989 aus der Hand der dänischen Königin Margrethe II. mit dem Kommandeurskreuz des dänischen Dannebrogordens ausgezeichnet wurde, verlieh seinem Verdienst als interkulturellem Mittler seinen besonderen Ausdruck.

Die politische Dimension, die Röhrig der Erwachsenenbildung immer wieder zuschrieb und deren Reflexion er dementsprechend als Aufgabe der Wissenschaften betrachtete, charakterisierte er im Zusammenhang mit dem Kongress wie folgt:

Hier wird aber nicht nur Geschichte wieder hochaktuell, sondern Pädagogik und Theologie, verbunden mit Politik, treten ein in den Raum von Verantwortung und Entscheidung für die Zukunft, werden über die historische Reflexion hinaus zur réflexion engagée im Sinne Flitners und zu 'lebendiger Wissenschaft' im Sinne Grundtvigs.

Spannt Röhrig hier einen Bogen zwischen geisteswissenschaftlicher Pädagogik und lebensweltbezogener wissenschaftlicher Reflexion im Grundtvigschen Sinne, offenbart sich einmal mehr sein bildungs- 
theoretisch begründeter Impetus, dem eine der Humanität verpflichtete Erwachsenenbildung zu folgen habe.

Mit Paul Röhrig verliert die deutsche Pädagogik ihren besten Kenner der Grundtvigschen Volkshochschule. Es dürfte in seinem Sinne sein, wenn der von ihm immer wieder angestoßene Bildungsdiskurs auch und gerade mit Blick auf Grundtvig fortgefuihrt würde. Die in Vorbereitung befindliche neue deutsche Grundtvig-Ausgabe könnte hierzu einen hilfreichen Beitrag leisten.

Wissenschaftliche Publikationen (Auswahl)

Röhrig, Paul (1988), "Geschichte des Bildungsgedankens in der Erwachsenenbildung und sein Verlust" in Zeitschrift 'für Pädagogik, vol. 34.

— (1989), Lebendige Erwachsenenbildung - Reflexionen über die Aktualität von N.F.S. Grundtvig, Dialog, 10, Bederkesa

— (1991), "Um des Menschen willen" Grundtvigs geistiges Erbe als Herausforderung für Erwachsenenbildung, Schule, Kirche und soziales Leben, Weinheim.

- (2005), "Der bildungstheoretische Ansatz in der Erwachsenenbildung" in Tippelt, R. (Hrsg.), Handbuch Erwachsenenbildung/Weiterbildung, Wiesbaden.

Röhrig, Paul und Oppermann, D. (1995), 75 Jahre Volkshochschule. Vom schwierigen Weg zur Humanität, Demokratie und soziale Verantwortung, Bad Heilbrunn. 